\title{
Applicability of the Fels questionnaire on physical activity of children in rural areas of Bosnia and Herzegovina
}

\section{Vedran Đido ${ }^{1 *}$, Aida Pilav1,2, Marijan Marjanović3 ${ }^{1}$, Jenny Phillips ${ }^{4}$, Deana Švaljug ${ }^{5}$, Sandra Boškovićs , Hadžan Konjo ${ }^{1}$, Đemil Omerović ${ }^{1}$}

${ }^{1}$ Faculty of Health Studies, University of Sarajevo, Sarajevo, Bosnia and Herzegovina, ${ }^{2}$ Institute for Public Health of Canton Sarajevo, Sarajevo, Bosnia and Herzegovina, "3Faculty of Health Care, University "Vitez" Travnik, Travnik, Bosnia and Herzegovina, ${ }^{4}$ School of Health and Education, Middlesex University, London, UK, ${ }^{5}$ Faculty of Health Studies, University of Rijeka, Rijeka, Croatia

\begin{abstract}
Introduction: Insufficient physical activity is one of the leading public health problems in the world, but also in Bosnia and Herzegovina. Modern civilization is characterized by a significant decrease in physical activity, and the number of people whose lifestyle can be called sedentary has never been higher, which is especially emphasized among children and adolescents. Aim of the study is to examine public health significance of physical activity on the occurrence and the degree of obesity in children and adolescents in primary and secondary schools and to determine the applicability of the Fels questionnaire on physical activity of children in rural areas of Bosnia and Herzegovina.
\end{abstract}

Methods: We used a transversal research method of a cross-sectional study at a 1-time point, and for obtaining results, we used the Fels physical activity questionnaire for children and measurement protocol.

Results: Two hundred and seventy-six primary and secondary school students in two cities participated in this survey. Respondents in Busovača are more physically active than their peers in Sarajevo. One-third of the total number of respondents is overweight and obese, and respondents in Sarajevo are significantly more nourished than their peers in Busovača. The Fels questionnaire is conditionally applicable, especially in rural areas.

Conclusion: This study confirmed that the Fels questionnaire for assessing the level of physical activity for children and young people, which is the general instrument for research of physical activity in children, is too generalized because it is based on a homogeneous urban population.

Keywords: Fels questionnaire; physical activity; children; rural areas

\footnotetext{
*Corresponding author: Vedran Đido, Faculty of Health Studies, University of Sarajevo, Sarajevo, Bosnia and Herzegovina, E-mail: vedran.djido@fzs.unsa.ba
}

Submitted: 07 September 2020/Accepted: 12 October 2020

DOI: https://doi.org/10.17532/jhsci.2020.1072

UNIVERSITY OF SARAJEVO FACULTY OF HEALTH STUDIES

\section{INTRODUCTION}

Globally, most children and adolescents are relatively physically inactive or mostly not active enough to meet the recommendations of minimum physical activity (1). The trend of physical inactivity is 
growing rapidly in most societies around the world. It is not the fact only for countries with high income, but this trend also increases in countries with low and medium income (2), which is the consequence of rapid economic development, and changes in living environment and habits over the last two decades $(3,4)$.

There is a worldwide agreement that children should participate in moderate to vigorous physical activity at least 60 minutes a day to achieve significant health benefits (5). Following this, regularly recommended physical activity can improve cardiorespiratory and muscle fitness, bone health, cardiovascular, and metabolic biomarkers and can reduce symptoms of anxiety and depression (6), as well as prevent overweight and obesity in children and young people (7).

Obesity occurs in the early years of life and affects a large number of people worldwide. Its effects are associated with a decrease in quality of life and increase in early mortality (8).

The school environment seems to be a promising setting for the implementation of health interventions because it encourages dynamic interaction between students, families, teachers, and healthcare professionals, providing favorable conditions for adopting healthy habits, especially physical activity (8).

Although the importance of physical activity is now well recognized, the promotion of physical activity is still fairly young field compared to other risk factors for mass chronic non-communicable diseases, such as malnutrition and diet or the usage of tobacco. However, in recent years, there has been an increasing number in evidence of effective policies for the promotion of physical activity. The World Health Organization (WHO) and the European Commission have published policy guidelines to support developing countries in the development of comprehensive and effective national policies for the promotion of physical activity (9).

To raise public awareness of the importance of regular physical activity in maintaining and promoting good health and well-being, the WHO launched a global initiative on May 10, 2002, to mark International Day of Physical Activity (10).

\section{METHODS}

This study involved 276 primary and secondary school students in two cities selected by a random sample of which $50.7 \%(F=140)$ were primary school students and 49.3\% $(\mathrm{F}=136)$ secondary school students. Those are the city of Sarajevo and Busovača which, by standards, represent the urban and rural environment in the context of Bosnia and Herzegovina. In Sarajevo, $122(44.2 \%)$ students were surveyed, and they belonged to the category of an urban environment, and in Busovača 154 $(55.8 \%)$ students were surveyed and they were categorized as of rural environment. The study included both genders, and respondents participated voluntarily and anonymously, with prior parental consent for minor children. The average age of the respondents was 13.17 years. In this study, we used a transversal research method of a cross-sectional study at the 1-time point, and for obtaining results, we used the Fels physical activity questionnaire for children (11), the measurement protocol and standardized international questionnaire for the assessment of physical activity levels for children and young people. In this study, we used anthropometric features that best reflect certain morphological features. Respondents were measured in physical and health education classes while being in the equipment needed to attend classes without associated footwear. We used the International Biological Program (12) protocol for longitudinal skeletal dimensionality, mass, and body mass index for children calculated according to World Obesity/ Policy and Prevention Federation. Descriptive and inferential statistical tests were used for all measured variables. Descriptive statistics with central dispersion parameters were processed, and the normality of the distribution was examined. In inferential statistics, students' $t$-test and ANOVA test were used to examine differences at the univariate and multivariate analysis of levels. Pearson's correlation test and multiple regression analysis were performed in the correlation study. Statistical data processing was done in IBM SPSS Statistics 22.0 program.

\section{Ethics approval}

This research was approved by the Ethics Committee of the Faculty of Health Studies of the University of Sarajevo under the approval number 04-7-114-6/13. 


\section{RESULTS}

The results presented in Table 1 show that 59.8\% of students in Sarajevo (urban area) and $72.7 \%$ of students in Busovača (rural area) have normal body mass. $25.4 \%$ of students in Sarajevo and $18.8 \%$ of students in Busovača are overweight. About 14.8\% of students in Sarajevo and $8.4 \%$ of students in Busovača have a high obesity rate. Although there were visible differences in numbers, the differences in the level of nutrition status of respondents concerning their place of residence were calculated, and it was determined that there was a statistically significant difference $(p<0.01)$. Statistical significance was also calculated according to the place of residence $(p=0.054)$ and the sample is uniform. There is no statistically significant difference in the number of respondents by place of residence.

The results in Table 2 show that most respondents living in Sarajevo (41.8\%) very often go to and from school on foot and/or by bicycle, and most students living in Busovača $(48.1 \%)$ very often go to and from school on foot and/or by bicycle. By examining the differences, it was found that there was no statistically significant difference in the respondents' answers to the question whether they go to and from school on foot and/or by bicycle concerning their place of residence.

Table 3 shows the physical activity indexes of respondents by place of residence. The results show that students in Busovača (rural area) are more physically active (8.19) than students in Sarajevo (urban area) (7.10) and that sports (3.26) and housework (3.06) account for the largest part of physical activity of students in Busovača.

TABLE 1. Nutrition status of the respondents (Sarajevo versus Busovača)

\begin{tabular}{llc}
\hline Residence & BMI $^{*}$ & $\mathrm{~F} \%$ \\
\hline Sarajevo & Normal body mass & 7359.8 \\
& Overweight & 3125.4 \\
& High obesity rate & 1814.8 \\
& Total (N) & 122100.0 \\
Busovača & Normal body mass & 11272.7 \\
& Overweight & 2918.8 \\
& High obesity rate & 138.4 \\
& Total (N) & 154100.0 \\
\hline
\end{tabular}

*Nutrition status based on calculated body mass index according to World Obesity/Policy and Prevention Federation
From the results shown in Table 4, we can see that the correlation coefficient between the respondents level of nutrition status and their place of residence is statistically negatively significant, which would mean that the level of nutrition status is better if the children live in a rural area (Busovača) and vice versa. The level of nutrition status is worse if the respondents are residents in an urban area (Sarajevo).

By calculating the relationship between the level of physical activity and the level of nutrition status in children and young people in primary and secondary schools, it was found that there was no statistically significant correlation. The results presented in the regression analysis table show that the Beta coefficient is 0.055 .

TABLE 2. How often do you walk and/or bicycle to and from school?

\begin{tabular}{lc}
\hline Residence & $\mathrm{F} \%$ \\
\hline Sarajevo & \\
Never & 2117.2 \\
Rarely & 119.0 \\
Sometimes & 2419.7 \\
Often & 129.8 \\
Very often & 5141.8 \\
Total (N) & 11997.5 \\
Busovača & \\
Never & 1811.7 \\
Rarely & 149.1 \\
Sometimes & 1912.3 \\
Often & 2516.2 \\
Very often & 7448.1 \\
Total (N) & 15097.4 \\
\hline
\end{tabular}

TABLE 3. Descriptive indicators of physical activity index by students in a rural and urban area

\begin{tabular}{llllll}
\hline $\begin{array}{l}\text { Physical } \\
\text { activity index }\end{array}$ & Place of residence & M & Min. & Max. & SD \\
\hline Sport index & Busovača & 3.26 & 0.67 & 5.00 & 0.78 \\
& Sarajevo & 2.97 & 0.67 & 4.67 & 0.81 \\
Free time & Busovača & 1.88 & 0.50 & 2.50 & 0.46 \\
index & Sarajevo & 1.77 & 0.50 & 2.50 & 0.48 \\
Housework & Busovača & 3.06 & 0.50 & 5.00 & 0.80 \\
index & Sarajevo & 2.36 & 0.50 & 4.50 & 0.82 \\
Total physical & Busovača & 8.19 & 1.17 & 12.00 & 1.43 \\
activity & Sarajevo & 7.10 & 4.17 & 10.17 & 1.44 \\
\hline
\end{tabular}


TABLE 4. Correlation coefficient between nutrition status and place of residence of children

\begin{tabular}{lcc}
\hline Pearson correlation coefficient & Residence & Nutrition status \\
\hline Residence & & \\
Pearson correlation coefficient & 1 & $-0.139^{*}$ \\
Level of significance & & 0.021 \\
N & 276 & 276 \\
Nutrition status & & \\
Pearson correlation coefficient & $-0.139^{*}$ & 1 \\
Level of significance & 0.021 & \\
N & 276 & 276 \\
\hline
\end{tabular}

${ }^{*}$ Correlation is significant at level $0.05 \%$.

\section{DISCUSSION}

Through a review of the available literature and a critical review, we noticed that there was no single approach, attitude, opinion, or methodology for determining the actual level of physical activity in the general population, and especially not in the population of school children. While some preferred subjective procedures in obtaining data on the level and degree of children's participation in physical activity, such as survey questionnaires, others used objective procedures such as accelerometers and pedometers which children wore during the research. However, both methods have their advantages and disadvantages.

On the one hand, accurate instruments for measuring physical activity are needed by both clinical and research communities. Furthermore, the "self-quantification" of health parameters in everyday life and health care is becoming increasingly popular, which further increases the demand for accurate devices. Accelerometry is the most used objective measurement of physical activity for children and adults (13). The biggest disadvantage of this approach is the cost, and therefore we did not use it in our research.

On the other hand, subjective options for assessing the physical activity of children and young people which are included in questionnaires, often in the form of self-assessments and surveys conducted independently or under the supervision of interviewers, are widely used. While these instruments can offer a large amount of data (quantitative and qualitative) in a highly structured and analyzed form, they differ in type (e.g., school, household, professional, transportation, leisure, and sports, i.e., mode of operation, time frame, frequency, etc.). These methods are attractive to researchers, primarily because of their practicality and convenience. Despite these advantages, questionnaires aimed at the demographic group of young people consistently show low or moderate correlation with objective measures, can provide answers based on social desirability, and are prone to excessive underestimation and misinterpretation, all of which contribute to reliability and validity (14).

To assess the level of physical activity of children and young people, we used a questionnaire called the Fels Questionnaire on Physical Activity for Children, which was standardized and widely used by many researchers around the world, especially in Europe (15).

Although we found out that the questionnaire was not fully applicable in our research, we consciously used it unchanged because, in the first place, it was internationally standardized and was used several times in many studies, which allowed us to compare these results with the results from our research.

Evidence of conditional applicability in our circumstances, especially for rural areas, lies in the results of the research we cited previously, especially in Table 2 (a very crucial indicator in our research although it is a descriptive indicator). Namely, due to the generality of the Fels questionnaire (closed-ended questions), we do not know the walking/cycling distance that children cross on the way to school and back, because it is not the same whether respondents walk to school and back for $200 \mathrm{~m}$ on a flat sidewalk (which is the case for the urban population in Sarajevo) or $2 \mathrm{~km}$ on an uneven, hilly, macadam road through a forest (which is a very common case for the rural population in Busovača). The reason for this conditional applicability is that the questionnaire is not differentiated. This is conditionally applicable, or rather the question with questionable validity for children living in rural areas, as children from rural areas are more physically active (Table 3), they walk more in different geographic landscapes on their way to school with significantly different walking/cycling distances to schools and have a significantly larger energy consumption as a result, as opposed to children who live in urban areas, where above-mentioned facts were virtually homogeneous. 
Furthermore, when asked "What tasks do you do at home that requires physical activity, and how often do you do them?" we received highly heterogeneous responses when we compare respondents living in urban and rural areas. While respondents in the urban area performed light physical tasks such as taking out household waste, vacuuming, dusting, and cleaning the room, respondents in a rural area mostly performed difficult physical tasks from cutting, splitting, and stacking firewood to hard works in agriculture and cattle breeding. We asked this question outside the official Fels Questionnaire because it is not differentiated nor offers generalized answers such as light housekeeping, medium-heavy household chores, and heavy household chores.

We analyzed all of these facts in the context of the same nutrition habits for respondents both in urban and rural areas, although we were aware of the fact that this was not the case; however, child nutrition habits were not the subject of our research, so we have no evidence to support this.

Due to heterogeneous conditions in which children live in rural and urban areas in our country, the Fels questionnaire becomes conditionally applicable, since it was created and standardized in urban environments and conditions that are homogeneous.

In 2015, Karakaš et al. conducted a study following the same protocol (Fels questionnaire) as we did, regarding physical activity in girls aged 7-11 in Canton Central Bosnia. The results on the level of physical activity according to the Likert scale showed that the largest number of girls who mainly engage in physical activity is 167 (57.39\%), while in the total sample there were no girls who did not engage in physical activity at all. Regarding the differences in the values of the sports index, the leisure index and the household index, physical activity is most emphasized during sports activities, while the lowest value of physical activity was recorded while doing household chores (16). We could not compare this research with ours because it had been performed only among girls and correlation in terms of place of residence (urban vs. rural environment) was not performed.

In 2011, Azarbayans et al. confirmed a negative correlation between BMI and physical activity $(p<0.025)$ (17), which did not correlate with our results. Regression analysis (Table 5) showed that the Beta coefficient is 0.055 .

Abreu, Nascimento, Jardim, and Rozov in 2010 were researching to determine the validity and reliability of APARQ, FELS, and YOUTH Questionnaires for Brazilian children, where intraclass coefficient correlation for the reliability of the Fels Questionnaire (among others) was determined and showed good reliability (18). We could not correlate this research to ours because we did not investigate the validity and reliability of the Fels Questionnaire, but it gave us insight into the reliability of the questionnaire for research purposes.

The Fels questionnaire on physical activity assessment of children and young people is moderately reliable for all age groups of children (19).

A 2011 study conducted in England confirmed that there was no difference in BMI between rural and urban children $(<13$ years) or adolescents $(>13$ years) (20) which was not consistent with our research, in which we found a statistically significant difference between urban and rural areas (Tables 1 and 4).

\section{Strengths and limitations}

The key strength of this study was that according to the literature research available to us, this was the only study that tested the applicability of the standardized Fels physical activity questionnaire and

TABLE 5. Relationship between physical activity and nutrition status in children and young people

\begin{tabular}{lccccc}
\hline ANOVA & Sum of squares & Df & MS & F & Level of significance \\
\hline Regression & 0.394 & 1 & 0.394 & 0.833 & \\
Residue & 129.678 & 274 & 0.473 & & \\
Total & 130.072 & 275 & & & 0.362 \\
\hline Coefficients & $\mathrm{B}$ & Standard M error & Beta & T & Level of significance \\
\hline Obesity level & 1.252 & 0.213 & & 5.886 & 0 \\
Physical activity level & 0.025 & 0.027 & 0.055 & 0.913 & 0.362 \\
\hline
\end{tabular}


compared its application in highly urban and rural areas, with evidence of the questionable applicability of the questionnaire in rural areas.

The limitation of the study is in the relatively small number of respondents and the non-inclusion of the entire country in the research. In the use of questionnaires as research tools, there was a possibility of non-objective data filling. It is also important to note that a number of respondents did not answer all the questions in the survey. All received answers were included in the results.

\section{CONCLUSION}

The correlation between total physical activity and level of nutrition status in children and young people in primary and secondary schools in the total population that is surveyed is statistically significant, especially in the context of the place of residence. One-third of the total surveyed population of children and young people in primary and secondary schools is overweight and has a high obesity rate, and there is a statistically significant difference in the context of the place of residence.

This study confirmed that the general instrument for the study of physical activity in children, Fels questionnaire on the assessment of physical activity level for children and young people, is too generalized because it is based on a homogeneous urban population, primarily in Western developed countries, where there are no significant differences between children in urban and rural areas, as opposed to children in Bosnia and Herzegovina, where there are significant differences in lifestyle, attitudes, behavior, and socioeconomic status between urban and rural areas. Therefore, the Fels questionnaire is conditionally applicable. It is recommended that the Fels questionnaire is expanded and specified in specific sections with sub-questions to be scaled and based on that the research should be expanded and repeated.

\section{ACKNOWLEDGMENTS}

This research was conducted with the support and devoted help of employees in two primary and two secondary schools in Central Bosnia Canton and Sarajevo Canton. Also, acknowledgements to Margarita Treuth, Ningqi Hou, Deborah Young and L. Michele Maynard who provided us with a questionnaire for this research.

\section{REFERENCES}

1. de Moraes AC, Guerra PH, Menezes PR. The worldwide prevalence of insufficient physical activity in adolescents a systematic review. Nutr Hosp 2013;28(3):575-84.

2. Muller AM, Khoo S, Lambert R. Review of physical activity prevalence of Asian school age children and adolescents. Asia Pac J Public Health 2013;25(3):227-38 https://doi.org/10.1177/1010539513481494.

3. $\mathrm{Xu} P$, Chen $\mathrm{Y}$, Ye $\mathrm{X}$. Haze, air pollution, and health in China. Lancet 2013;382(9910):2067. https://doi.org/10.1016/s0140-6736(13)62693-8.

4. Congchao L, Stolk R, Sauer P, Sijtsma A, Wiersma R, Huang G, et al Factors of physical activity among Chinese children and adolescents: A systematic review. Int J Behav Nutr Phys Act. 2017;14:110.

5. Gába A, Dygrýn J, Mitáš J, Jakubec L, Frömel K. Effect of accelerometer cut-off points on the recommended level of physical activity for obesity prevention in children. PLoS One 2016;11(10):1-15 https://doi.org/10.1371/journal.pone.0164282.

6. World Health Organization. Global Recommendations on Physical Activity for Health. Geneva: World Health Organization; 2010. Available from: http://www.who.int/dietphysicalactivity/factsheet_recommendations/en. [Last accessed on 2019 Jun 03]

7. Sigmund E, Sigmundova D, Badura $P$, Kalman M, Hamrik Z, Pavelka J. Temporal trends in overweight and obesity, physical activity and screen time among Czech adolescents from 2002 to 2014: A national health behaviour in school-aged children study. Int J Environ Res Public Health 2015;12(9):11848-68. https://doi.org/10.3390/ijerph120911848.

8. Todaro R. Handbook of Physical Education Research: Role of School Programs, Children's Attitudes and Health Implications. New York: Nova Science Publishers, Inc.; 2014.

9. Kahlmeier S, Wijnhoven TM, Alpiger P, Schweizer C, Breda J, Martin BW. National physical activity recommendations: Systematic overview and analysis of the situation in European countries. BMC Public Health 2015;15:133 https://doi.org/10.1186/s12889-015-1412-3.

10. Institute for Public Health of Republic of Srpska. International Day of Physical Activity. Available from: http://www.phi.rs.ba/index.php?view=clanak\&id=247. [Last accessed on 2019 Jul 13].

11. Treuth M, Hou N, Young D, Maynard LM. Fels PAQ for children. Available from: https://www.measurement-toolkit.org/pdf/pa/FELS_PAQ_FOR_ CHILDREN.pdf. [Last accessed on 2020 Oct 01].

12. Weiner J, Lourie J, International Biological Programme. Human Biology, A Guide to Field Methods. Ipswich, MA: ERIC; 1969.

13. Hildebrand $M$, Hansen $B H$, van Hees VT, Ekelund $U$ Evaluation of raw acceleration sedentary thresholds in children and adults. Scand J Med Sci Sports 2016;27(12):1814-23 https://doi.org/10.1111/sms.12795.

14. Wójcicki T, McAuley E. II. Physical activity: Measurement and behavioral patterns in children and youth. Monogr Soc Res Child Dev. 2014;79(4):7-24. https://doi.org/10.1111/mono.12128.

15. Chinapaw MJ, Mokkink LB, van Poppel MN, van Mechelen W Terwee CB. Physical activity questionnaires for youth: A systematic review of measurement properties. Sports Med 2010;40(7):539-63. https://doi.org/10.2165/11530770-000000000-00000.

16. Karakaš S, Osmani Z, Paklarčić M, Kukić E. Analiza preferencije i 
učestalosti bavljenja tjelesnom aktivnošću kod djevojčica uzrasta 7-14 godina sa područja Srednjobosanskog Kantona. J Anthropol Soc Serbia 2015;50:17-24.

17. Azarbayjani M, Tojari F, Habibinejad M. The relationship between obesity, physical activity and socioeconomic status among girl students living in Northern Tehran. Feyz J Kashan Univ Med Sci 2011;15(2):132-8.

18. Abreu LR, Nascimento OA, Jardim JR, Rozov T. Reliability and validity of the adolescent physical activity recall questionnaire (APARQ), the fels physical activity questionnaire (FELS) and the youth risk behavior survey (only physical activity part-YOUTH) in
Brazilian children. Am J Respir Crit Care Med. 2010;181:A3465. https://doi.org/10.1164/ajrccm-conference.2010.181.1_meetingabstracts. a3465.

19. Treuth M, HouN, Young D, MaynardL. Validityand reliability of the fels physical activity questionnaire for children. Med Sci Sports Exerc 2005;37(3):488-95. https://doi.org/10.1249/01.mss.0000155392.75790.83.

20. Sandercock G, Ogunleye A, Voss C. Comparison of cardiorespiratory fitness and body mass index between rural and urban youth: Findings from the East of England healthy hearts study. Pediatr Int 2011;53(5):718-24. https://doi.org/10.1111/j.1442-200x.2011.03354.x. 